\title{
LES ÉTUDES CONTRASTIVES ET CONFRONTATIVES: DOMAINE FRANÇAIS-POLONAIS
}

Abstract. Stefański Witold, Les études contrastives et confrontatives: domaine français-polonais [Contrastive and confrontative Studies: French-Polish], Studia Romanica Posnaniensia, Adam Mickiewicz University Press, Poznań, vol. XXVII: 2001, pp. 153-156. ISBN 82-232-1039-X, ISSN 0137-2475.

In this article problems of contrastive and confrontative studies in reference to Polish and French is discussed. The author also deals with the relationship of these studies with typological linguistics. The discussion is based on analyses of oppositions which make it possible to classify languages into analytical and synthetic ones.

Si nous avons choisi ce sujet c'est pour deux raisons: la permière est le fait que Poznań est devenu un centre de recherches contrastives et confrontatives. Il suffit de rappeler le nom de Ludwik Zabrocki, qui a beaucoup contribué à cette discipline et la revue Papers and Studies in Contrastive Linguistics qui est publiée à Poznań. Deuxièmement, le professeur Stanisław Gniadek, qui travaillait à l'Université de Poznań, a publié Grammaire contrastive franco-polonaise (1979).

Le terme de contrastive apparaît dans les dictionnaires dans de divers contextes. Dans Encyklopedia jezykoznawstwa ogólnego (1995), nous le trouvons sous l'entrée gramatyka kontrastywna («grammaire contrastive»), dans le dictionnaire allemand de Bussmann (1990), sous kontrastive Linguistik («linguistique contrastive»). On peut donc parler d'une approche, d'une méthode, d'une grammaire, et même d'une linguistique contrastive. Nous voudrions essayer de trouver une place pour la linguistique contrastive parmi les disciplines de la linguistique puisque dans les dictionnaires on mentionne plusieurs variantes de la linguistique, qu'on est amené à se demander, s'il existe plusieurs linguistiques ou plutôt une seule avec plusieurs perspectives de recherches.

Personnellement, nous sommes d'avis qu'il n'y a qu'une seule linguistique, mais que plusieurs perspectives d'investigation sont possibles. La plus importante et la plus ancienne est la linguistique descriptive qui a donné naissance, à la suite de la 
multiplication d'objets de description, à la linguistique comparative. Il existe aujourd'hui deux variantes de cette linguistique:

1) la linguistique historico-comparative (=linguistique comparée),

2) la linguistique synchrono-comparative.

L'analyse synchrono-comparative idéale devrait donner comme résultat une description complète et exhaustive de deux ou plusieurs langues. Pour des raisons didactiques on distinguait des traits divergents, du domaine de la linguistique contrastive et des traits convergents, appartenant au domaine de la linguistique confrontative. Comme dans plusieurs autres cas, on distingue les analyses totales et partielles. On pourrait admettre une équation suivante pour l'analyse comparative totale: grammaire synchrono-comparative $=$ grammaire contrastive + grammaire confrontative.

De cette manière il nous est maintenant plus facile de comprendre les liens qui unissent la grammaire contrastive-confrontative avec la linguistique typologique qui étudie autant les différences que les ressemblances entre les langues. Pour cela les typologues utilisent depuis longtemps les termes de synthétique et d'analytique qui furent introduits pour la première fois par Auguste Guillaume Schlegel (1767-1845) dans sa monographie Observations sur la langue et la littérature provençales (1818).

Notre présent article a pour but de démontrer l'utilité de ces deux termes pour définir les caractéristiques du français et du polonais. Le premier problème concerne l'extension de sens des termes: synthétique et analytique. Nous savons que dans les manuels de linguistique on exemplifiait ces phénomènes par la structure du groupe nominal et verbal. Si nous comparons la forme française à la fille avec la forme polonaise dziewczynie (Dat. sg.), il est clair que les catégories de cas, genre et nombre sont exprimées en français à l'aide de morphèmes détachés, d'une façon analytique alors, tandis que dans la langue polonaise ces catégories sont exprimées d'une façon synthétique par la terminaison flexionnelle.

La tâche de la linguistique consiste à établir des liens logiques entre les phénomènes de base des rapports cause-effet. Dans les dictionnaires de linguistique on lit que la différenciation synthétique / analytique concerne les niveaux morphologique et syntaxique. Le niveau phonétique / phonologique, dépourvu de sens est rejeté et est traité à part. Or, il nous semble que les rapports entre les niveaux d'analyse linguistique ne sont suffisamment connus. Aujourd'hui, nous sommes d'avis que le phénomène d'être synthétique ou analytique (qu'il s'agisse d'un type ou d'une tendance) commence au niveau phonético-phonologique. Aussi le caractère synthétique du polonais est confirmé à ce niveau-là par les phénomènes suivants:

1) groupes consonantiques complexes,

2) alternances consonantiques (morphonologie),

3) corrélation des consonnes dures et mouillées.

De même que l'abscence de ces phénomènes en français prouve le caractère analytique de cette langue. 
Ce phénomène est aussi visible au niveau morphologique. Nous savons que la morphologie comprend la flexion et la formation des mots. Nous avons déjà montré le caractère synthétique de la flexion nominale polonaise et son caractère analytique en français. En ce qui concerne la flexion verbale, nous savons que la personne est exprimée en français d'une façon générale à l'aide de formes pronominales détachées tandis qu'en polonais à l'aide des terminaisons flexionnelles. Nous y reviendrons dans la suite de nos réflexions.

Dans le domaine de la formation des mots au moins deux arguments confirment le caractère analytique du français. Tout d'abord, en ce qui concerne les composés, il faut rappeler qu'en polonais le type synthétique est naturel, p.ex. francusko-polski ou dlugowlosy, en français pourtant le type franco-polonais est plutôt recessif, les constructions analytiques comme français-polonais et aux longs cheveux sont utilisées. Quant à la dérivation, on observe la disparition des suffixes diminutifs en français. Ainsi la forme ancienne fillette est remplacée par une construction analytique petite fille. Par contre, comme nous le savons, en polonais on note une quantité de suffixes diminutifs, p. ex. córka - córeczka, córusia, córuchna, córeńka etc. Les formes diminutives peuvent être considérées comme une comparaison interne. Or, aussi la catégorie de comparaison (= comparaison externe) est exprimée en français d'une façon analytique, p. ex. fort - plus fort - le plus fort, tandis qu'en polonais l'ancien type synthétique persiste: silny - silniejszy - najsilniejszy. Il faut pourtant ajouter que des restes des formes synthétiques fonctionnent en français, p.ex. bon-meilleur, et que, comme celui-ci, le polonais connaît aussi des constructions analytiques, p.ex. słodszy - bardziej słodki „plus doux". Certains linguistes au lieu de parler des types synthétique et analytique préfèrent le terme de tendance synthétique et analytique dans les langues.

Si nous faisons référence à la linguistique typologique, on distingue un aspect morphologique (type synthétique) et un aspect syntaxique (type analytique). L'aspect qui est étroitement lié à la catégorie de temps a un caractère synthétique en polonais et analytique en français: pol. pisz - napisz / fr. j'écris - j'aurai écrit ou pol. pisatem - napisatem / fr. j'écrivais - j'avais écrit. Dans le domaine de la diathèse, on voit le type analytique dominer aussi bien en français qu'en polonais. Les causatifs pourtant sont encore des formes synthétiques en polonais, analytiques déjà en français: pić - poić / boire - faire boire. Comme nous le voyons presque toutes les catégories morpho-syntaxiques sont exprimées d'une manière analytique en français et plutôt d'une façon synthétique en polonais.

Enfin, au niveau syntaxique, la nature synthétique du polonais se traduit par l'ordre des composants de la phrase libre et le régime entre le verbe et l'objet direct, p.ex. Chtopiec widzi psa (Gen.) - Le garçon voit un chien. Dans le groupe nominal, l'emploi des cas synthétiques oppose le polonais au français, p.ex. dom brata (Gen.) - la maison du frère.

Ainsi dans notre travail, nous avons envisagé tous les niveaux d'analyse linguistique, le niveau phonético-phonologique inclus, en démontrant les rapports logiques entre ce niveau et les niveaux morpho-syntaxiques. Nous avons montré 
pourquoi le polonais est une langue synthétique et pourquoi le français appartient au type de langue analytique. Nous avons aussi voulu montrer comment la perspective typologique peut enrichir les études contrastives.

\section{BIBLIOGRAPHIE}

B ussmann H. (1990), Lexikon der Sprachwissenschafi, Stuttgart: Kröner.

Duboi s J. et al. (1994), Dictionnaire de linguistique et des sciences du langage, Paris: Larousse.

Encyklopedia językoznawstwa ogólnego, sous la red. de Kazimierz Polański, Wrocław - Warszawa Kraków: Ossolineum 1995.

Gni adek St. (1979), Grammaire contrastive franco-polonaise, Warszawa: PWN.

Heinz A. (1978), Dzieje jezzykoznawstwa w zarysie, Warszawa: PWN. 